\title{
28 Research Soure \\ The Asbestos Contamination of Body Powder And Its Effect On Ovarian Health
}

\author{
Yin, YS \\ Guangdong Pharmaceutical University \\ Liu, HY ( $D$ liuhuanyu03@163.com ) \\ Guangdong Pharmaceutical University
}

\section{Research Article}

Keywords: Body powder, Asbestos, Ovary

Posted Date: February 4th, 2022

DOI: https://doi.org/10.21203/rs.3.rs-1237040/v1

License: (c) (i) This work is licensed under a Creative Commons Attribution 4.0 International License. Read Full License 


\section{Abstract}

Objective Investigation of asbestos pollution in body powder, and to explore the influence of asbestos contamination of body powder on ovary.

Methods X-ray diffraction combined with polarized light microscopy was used to detect asbestos in body powder sold offline and online in China. Forty-two female SD rats were distributed into the control group, the talc group, the asbestos group, and the asbestos+talc group $0.2 \mathrm{ml}$ corresponding powder suspension of $125 \mathrm{mg} / \mathrm{ml}$ concentration was injected through the reproductive tract once a day for 180 days. At 90 and 180 days, CA125 and HE4 were measured, and the ovaries of rats were taken for pathological sections.

Results A total of 13 asbestos samples were detected in 193 samples. The asbestos detection rate of online samples was higher than that of offline samples. The asbestos detection rate of samples in prices less than 10 Chinese Yuan was the highest. At 90 days and 180 days, the levels of CA125 and HE4 in each experimental group were increased, and the HE4 in the asbestos+talc group was higher than that in the asbestos group and talc group.At 180 days, the CA125 and HE4 levels of experimental groups were higher than those at 90 days. At 180 days, in the cortical area of rats in the asbestos+talc group, granular cell dysplasia was found in the follicles, and pathological mitotic figures were observed.

Conclusion Powder mixture of talc and asbestos in low concentration entering the body through the reproductive tract in long time can damage ovarian health.

\section{Introduction}

Johnson \& Johnson has been widely discussed for the huge compensation to the cancer caused by the use of Johnson \&Johnson's body powder in recent years. Several women have reportedly suffered from ovarian cancer due to long-term use of Johnson \&Johnson 's body powder, and the FDA announced in 2019 that Johnson \& Johnson's body powder contained trace asbestos. ${ }^{\text {. }}$

Body powder is a common cosmetics that can clean the skin and effectively absorb sweat to keep the skin dry and smooth. The main components of most body powder are talcum powder and essence. However, talcum often coexists with serpentine containing asbestos under natural conditions. If asbestos in talc is not strictly separated, it may lead to asbestos pollution of body powder. Asbestos is a class 1 carcinogen announced by the International Agency for Research on Cancer. Studies have found that asbestos fiber has obvious cytotoxicity on $\mathrm{CHO}$ cells. ${ }^{\square}$ Graham ${ }^{\natural}$ found that the epithelial cells of guinea pigs and rabbits injected with asbestos intraperitoneally have papillary changes, similar to those of patients with early ovarian cancer. There are few studies on the effects of asbestos on the ovarian health domestically and abroad. Human ovaries are usually not directly exposed to asbestos. However, Hendesron ${ }^{\square}$ proved that talcum powder can be attached to the ovary and uterus by vaginal injection in rats, and Johnson ${ }^{\square}$ also found talcum particles in the resected tissues of ovarian cancer patients, which 
have the similar size and shape with those of infant body powder containing talcum powder. Therefore, if the is contaminated by asbestos, as asbestos fibers with talc powder enter the vagina and cervix through the vulva, asbestos may adhere to the surface of the ovary, and continuous stimulation may lead to the proliferation of ovarian epithelial cells, which may induce ovarian diseases and even ovarian cancer.

In this study, the body powders sold online and offline in Zhongshan City, Guangdong Province were sampling inspected, and the asbestos composition in body powder was determined by X-ray diffraction combined with polarized light microscopy to understand the asbestos pollution status of body powder. At the same time, through exploring the effect on the ovarian health of rats by injecting a small amount of talc or asbestos powder suspension into the reproductive tract of SD rats, the possible ovarian health damage caused by asbestos contamination of body powder in real life was explored.

\section{Materials And Methods}

\section{Sampling survey to detect asbestos pollution in body powder}

The offline sampling of Zhongshan City in Guangdong Province is divided into districts according to geographical distribution (Shiqi District, East District, West District, South District, Wugui Mountain Area, Torch Development Zone). Two locations are randomly selected from each district, namely large supermarkets and retail wholesale markets. 1-3 samples of different brands from different shops are randomly selected from each sampling site. Online sampling is randomly selected from Pinduoduo and Taobao.

The asbestos detection method was as follows : $10 \mathrm{~g}$ of body powder sample was weighed, ground to 300 mesh, sieved. The sample was ashed at $450^{\circ} \mathrm{C}$ in a muffle furnace(Nabertherm) for an hour. The ashed sample was pressed vertically in glass plate groove under appropriate pressure, and the groove was placed upwards in an X-ray diffractometer(RigakuMiniFlex 600) for detection. The Measurement condition of the X-ray diffractometer was as follows: scan range $5^{\circ}-64^{\circ}$, step width $0.02^{\circ}$, and scan speed $2^{\circ} / \mathrm{min}$. If there is no asbestos mineral diffraction peaks in the results, it is determined that the sample does not contain asbestos. For the samples with diffraction characteristic peaks of asbestos minerals, an appropriate amount of refractive index liquids(Cargille) of 1.550 was added to the glass slide. The wet sample particles were fully dispersed to avoid particle aggregation, and the glass slide was covered. The samples were observed under a polarizing microscope(OLYMPUS CX31-P). If fiber minerals with aspect ratio greater than 3 are found, it is determined that the sample contains asbestos.

\section{Animal toxicological experiments of talcum powder and asbestos}

Forty-two female SD rats(8-10 weeks old, weight 200-220g) were purchased from the Guangdong Medical Lab Animal Center(Guangzhou,China). All rats were raised at the Guangdong Pharmaceutical University 
Undergraduate Laboratory Animal Center(Guangzhou,China), temperature $20-26^{\circ} \mathrm{C}$, humidity $40 \%-70 \%$, $12 \mathrm{hours}$ alternating light and dark, free eating, and adaptive feeding for a week. The entire study protocol was carried out in accordance with the ethical principles defined by the Guangdong Pharmaceutical University for Animal Experimentation. The entire study is approved by Guangdong Pharmaceutical University for Animal Experimentation ethics committee. The ethical code for this experiment is gdpulac spf2017433. All methods are reported in accordance with ARRIVE guidelines

Normal saline was used as the medium to prepare talc powder(Tianjin Zhiyuan Chemical Reagent Co.,Ltd.)suspension, asbestos powder $\square$ Chrysotile asbestos is collected from Mangya asbestos mining area in Qinghai, China.【suspension and talc-asbestos mixed powder suspension by mixing with talc powder and chrysotile powder which were ground to 300 meshes. SD rats were randomly distributed into asbestos group ( $n=11)$, talc group $(n=10)$, asbestos+talc group $(n=11)$ and control group $(n=10) .1 \mathrm{ml}$ disposable syringe was inserted into the vaginal orifice of rats. Each rat in the experimental group was injected with $0.2 \mathrm{ml}(125 \mathrm{mg} / \mathrm{ml})$ corresponding suspension, and each rat in the control group was injected with $0.2 \mathrm{ml}$ normal saline,once a day, observe the animal behavior. After 90 days, 4 rats in each group were randomly selected to dissect bilateral ovaries. The tissue was washed in normal saline and fixed with $10 \%$ formalin for 24 hours, then embedded in paraffin and cut into $5 \mu \mathrm{m}$ thickness. Then they were processed and stained with hematoxylin-eosin and observed under optical microscope for morphological evaluation. About $1 \mathrm{~mL}$ of rat tail vein blood was taken and centrifuged at $1000 \mathrm{r} / \mathrm{min}$ for $20 \mathrm{~min}$ to separate serum. The levels of CA125 and HE4 were determined by ELISA (the specific operation was carried out according to the steps described in the kit. The kit was purchased from Huangshi Research Biotechnology Co., Ltd.). After 180 days, the same methods were used on the remaining rats for pathomorphological analysis and detection of CA125, HE4.

\section{Statistical Analysis}

SPSS26.0(IBM Corporation,Armonk,NY,USA) for data processing. Chi-square test is used for the comparison of rates. If the theoretical frequency $\mathrm{T}$ in the RxC contingency table is less than 1 , Fisher exact probability method is used. Through the normality test, it was found that the body weight, ovarian weight, serum CA125 and HE4 of rats in this study were all in normal distribution, and the description was expressed as mean \pm standard deviation. One-way analysis of variance was used for comparison among groups. SNK test was used for further comparison between groups. Paired $t$ test was used for comparison of the same measurement index at different experimental stages. Differences were considered statistically significant when $\mathrm{P}<0.05$.

\section{Results}

\section{The detection results of asbestos contamination in body powder}


It was found that there were 13 diffraction peaks of asbestos minerals in 193 samples(Figure 1), and further polarizing microscope observation showed that all the 13 samples contained asbestos fibers(Figure 2). The qualified rate of online samples was lower than that of offline samples $(X 2=4.045$, $\mathrm{P}<0.05)$. In addition, Sampling inspection showed that for the same brand, asbestos was detected in the samples extracted from online route, while not detected from offline route. There was no significant difference in asbestos test results of refreshing powder purchased from different offline routes $(X 2=$ $0.775, P>0.05)$. There was no significant difference in asbestos test results among different online purchases $(\chi 2=0.706, P>0.05)($ Table 1$)$.

Table 1

detected results of asbestos in body powder samples from different sampling sites

\begin{tabular}{|llll|}
\hline Sampling sites & Number of samples & Number of unqualified & Qualified rate,\% \\
\hline offline & 81 & 2 & 97.53 \\
\hline Shopping malls & 45 & 0 & 100 \\
\hline Retail,wholesale markets & 36 & 2 & 94.44 \\
\hline online & 112 & 11 & 90.18 \\
\hline Taobao & 49 & 3 & 93.88 \\
\hline Pinduoduo & 63 & 8 & 87.30 \\
\hline total & 193 & 13 & 93.26 \\
\hline
\end{tabular}

The difference of asbestos detection results of body powders in different prices was statistically significant $(X 2=11.349, P<0.05)$, and the asbestos detection rate of body powder samples with prices lower than 10 Chinese yuan was higher(Table 2).

Table 2

detected results of asbestos in samples with different prices of body powder

\begin{tabular}{|llll|}
\hline Price,Chinese Yuan & Number of samples & Number of unqualified & Qualified rate,\% \\
\hline$<10$ & 72 & 11 & 84.72 \\
\hline $10-20$ & 76 & 1 & 98.68 \\
\hline $20-30$ & 38 & 1 & 97.37 \\
\hline$>30$ & 7 & 0 & 100 \\
\hline total & 193 & 13 & 93.26 \\
\hline
\end{tabular}

\section{Weight changes of rats and bilateral ovarian wet weight}

At the different stages of the experiment, there was no significant difference in weight of rats in each group $(P>0.05)$. Compared with the control group, at 90 days, the ovarian wet weight of the asbestos 
group and the asbestos+talc group was higher than that of the control group $(P<0.05)$. There was no significant difference in ovarian wet weight between talc group and control group $(P>0.05)$. There was no significant difference in the ovarian wet weight among the asbestos group, the talc group and the asbestos+talc group $(P>0.05)$. At 180 days,the ovarian wetness of the asbestos+talc group was significantly higher than that of the control group $(P<0.05)$. There was no significant difference in ovarian wet weight among other groups $(P>0.05)$ (Table 3$)$.

Table 3

Weight and wet weight of ovary in each group(mean \pm SD)

\begin{tabular}{|llllll|}
\hline Group & Weight,g & & & \multicolumn{2}{l|}{ ovarian weight, g } \\
\cline { 2 - 6 } & $\begin{array}{l}\text { Before } \\
\text { exposure }\end{array}$ & Day $\mathbf{9 0}$ & Day 180 & Day 90 & Day 180 \\
\hline asbestos group & $222.26 \pm 9.89$ & $327.37 \pm 28.01$ & $371.30 \pm 46.14$ & $0.17 \pm 0.036$ & $0.13 \pm 0.029$ \\
\hline talc group & $220.52 \pm 7.66$ & $328.76 \pm 26.63$ & $379.80 \pm 63.57$ & $0.13 \pm 0.036$ & $0.13 \pm 0.014$ \\
\hline $\begin{array}{l}\text { asbestos+talc } \\
\text { group }\end{array}$ & $220.01 \pm 10.83$ & $346.79 \pm 15.37$ & $404.33 \pm 40.17$ & $0.16 \pm 0.039$ & $0.14 \pm 0.017$ \\
\hline $\begin{array}{l}\text { control group } \\
\text { Changes in Serum CA125 and HE4 levels of rats in each } \\
\text { group }\end{array}$ & $221.69 \pm 9.33$ & $347.65 \pm 11.48$ & $388.00 \pm 38.04$ & $0.09 \pm 0.014$ & $0.11 \pm 0.021$ \\
\hline
\end{tabular}

At 90 and 180 days, compared with the control group, the levels of CA125 and HE4 in each experimental group were increased $(P<0.05)$. There was no significant difference in CA125 level among asbestos group, talc group and asbestos+talc group $(P>0.05)$. There was no significant difference in HE4 levels between asbestos group and talc group $(P>0.05)$. HE4 of asbestos+talc group was higher than asbestos group and talc group $(\mathrm{P}<0.05)$.

At 180 days, the CA125 levels in the control group and each experimental group were higher than those at 90 days $(P<0.05)$. At 180 days, in each experimental group were higher than that at 90 days of experiment $(P<0.05)$, but there was no significant difference in the level of HE4 in the control group $(P>0.05)($ Table 4$)$ 
Table 4

Serum CA125 and HE4 levels of rats in each group (mean \pm SD)

\begin{tabular}{|lllll|}
\hline Group & Day 90 & \multicolumn{3}{c|}{ Day 180} \\
\cline { 2 - 5 } & CA125,U/ml & HE4,pmol/I & CA125,U/ml & HE4,pmol/I \\
\hline asbestos group & $9.65 \pm 1.46$ & $72.06 \pm 14.69$ & $10.88 \pm 1.51$ & $75.90 \pm 17.64$ \\
\hline talc group & $9.81 \pm 1.16$ & $71.71 \pm 13.66$ & $10.79 \pm 1.25$ & $73.76 \pm 12.14$ \\
\hline asbestos+talc group & $10.63 \pm 1.81$ & $91.75 \pm 14.82$ & $12.08 \pm 1.16$ & $95.57 \pm 15.34$ \\
\hline control group & $6.48 \pm 1.17$ & $39.84 \pm 9.81$ & $7.62 \pm 1.07$ & $45.53 \pm 6.83$ \\
\hline
\end{tabular}

\section{Pathomorphological analysis of ovarian tissue in rats}

At 90 days, the naked eye observation found that the ovaries of rats in the experimental group appeared different degrees of congestion and swelling, and two rats in the asbestos+talc group found uterine edema and a small amount of ascites, but the HE staining results of the ovarian tissue of rats showed only slight congestion and bleeding, and there was no obvious morphological and pathological changes of the ovary, so it was considered that the morphological results of the control group and the experimental group were not significantly different. At 180 days, the ovarian structure of the control group was normal, and there was no abnormal pathological change. The naked eye observation in the experimental group showed that the ovaries of rats had different degrees of congestion and swelling, and there were different degrees of uterine edema and ascites. The ovarian epithelial cells and follicles of rats in the talc group and asbestos group showed morphological changes, and multiple atresia follicles appeared. In the cortical area of rats in the asbestos+talc group, granular cell dysplasia was found in the follicles, and pathological nuclear fission images were visible(Figure 3)

\section{Discussion}

There is a regular array of atoms or ions in the crystal. X-ray diffraction method uses a certain wavelength of X-ray to irradiate on different crystals, and the $\mathrm{X}$-rays scattered by different atoms interfere with each other. The distribution direction and intensity of the X-ray diffraction in the crystal space are different, and the unique map of each mineral will be formed, so as to analyze the composition of the measured sample according to the comparative analysis of the obtained map. The method has the advantages of no damage to the sample, good repeatability, fast and effective, large amount of information and simple analysis. Combined with polarized light microscopy, it can further confirm the existence of asbestos fiber in powder cosmetics and improve the accuracy of detection. [ii]]

The detection rate of chrysotile powders with price less than 10 yuan and those purchased online was higher. This may be because manufacturers of cheap body powder are more likely to choose inferior talc raw materials in order to reduce costs, and do not pay attention to product quality control. In addition, due 
to the current regulatory system of China 's online shopping is not perfect, and the supervision is not enough, the unscrupulous merchants are more likely to sell the body powder of poor quality.

CA125 in this study is a high molecular weight glycoprotein antigen derived from luminal epithelial cells. As an important ovarian cancer-related antigen, CA125 is the main indicator of ovarian cancer screening, diagnosis, disease monitoring, prognosis and efficacy evaluation in clinic. [iii] HE4 is a newer tumor marker than CA125, which is highly expressed in the genital tract and epithelial cells of ovarian cancer tissues. It has high sensitivity and specificity in the early diagnosis of ovarian cancer, and can be used as a reliable sensitive indicator of ovarian cancer. [iii]-9[iv] 9999999$]$ Combined detection of serum CA125 and HE4 levels can effectively improve the sensitivity, specificity and accuracy of ovarian cancer detection. [Iv]]

At 90 days, CA125 and HE4 in each experimental group were significantly higher than those in the control group, but no obvious pathological changes were found in the ovarian tissue, suggesting that although the ovarian function of rats in the experimental group had changed, the ovarian structure had not been seriously damaged. At 180 days, the ovarian structure also changed significantly. In addition, The structural changes of ovaries in asbestos+talc group were also the most obvious.

It can be seen that either talc or asbestos dust, long-term low concentration exposure will affect ovarian health. Also indicating that if the powder was contaminated by asbestos, and if the user was too close to the genitals and exposed to the mixed dust for a long time, the impact on ovarian health was more serious. In addition, the infant's skin barrier function was incomplete, and the skin was thinner than that of adults, the keratinocytes were small, the body surface area was small, the sweat gland density was higher than that of adults, and the sweating temperature was higher than that of adults. The retention of sweat in the skin wrinkles caused irritation to the skin, and it was more likely to cause skin problems such as redness and prickly heat. [[vi]-[vii][viii]] So many parents used body powder for infants and children, and it was more frequently used in the thigh root, buttock and other parts that were prone to accumulate sweat. Children, especially infants' immune system and other physical functions are not fully developed. If the long time exposure to asbestos pollution near the genital area, the harm is more serious.

Some studies have found that direct exposure to talcum powder in the ovary can increase the expression of Gsr, Sod1 gene and miRNA, which is harmful to ovarian tissue $\left[{ }^{[i x]]}\right.$ Hamilton $[[x]]$ and so on injected talcum powder suspension into the ovary of rats, and the ovary formed obvious cysts and papillary changes. In addition, talcum powder can induce changes in key oxidoreductases, and enhance the prooxidation state of normal cells and epithelial ovarian cancer cells, and talc exposure can significantly induce cell proliferation of cancer cells. ${ }^{[[x i]]}$ Epidemiological studies and META analysis also show that there is a link between the use of talcum powder in genitalia and serous ovarian cancer. ${ }^{[[x i]-[x i i j][x i v]]}$ Many studies have confirmed in different ways that talcum powder has a certain impact on ovary health, but there is still a lack of research on the impact of asbestos on these aspects. In this study, animal experiments were carried out to verify the damage of talcum powder to ovarian health, which supplemented the impact of asbestos on ovarian health and the damage of asbestos pollution to ovarian health. 


\section{Declarations}

\section{Data Availability}

The datasets generated during and/or analysed during the current study are available from the corresponding author on reasonable request.

\section{Acknowledgement}

Thanks are due to HuanYu Liu for assistance with the experiment

\section{Conflicts of interest statement}

All authors disclosed no relevant relationships.

\section{Source of funding statement}

None declared

\section{Data availability statement}

All data generated or analysed during this study are included in this published article [and its supplementary information files].

The original data of rats weight and ovarian wet weight were not saved due to the damage of my computer. The original data of blood indexes were in the supplementary documents, and the original data of sampling survey were in the manuscript.

\section{A statement to confirm that all experimental protocols were approved by a named institutional and/or licensing committee[}

The entire study protocol was carried out in accordance with the ethical principles defined by the Guangdong Pharmaceutical University for Animal Experimentation.

Ethics number囚gdpulac spf2017433

the statement in the Methods section of my manuscript.

\section{Reference}

1. Dyer, O. Johnson \& Johnson recalls its baby powder after FDA finds asbestos in sample. BMJ. 2019,367,16118. 
2. Kenne, K., Ljungquist, S., Ringertz, N. R. Effects of asbestos fibers on cell division, cell survival, and formation of thioguanine-resistant mutants in Chinese hamster ovary cells.[J].Environmental Research.1986,39(2),448-464

3. Graham, J., Graham, R. Ovarian cancer and asbestos.[J]. Environmental Research.1967,1(2),115128.

4. Henderson, W. J. et al. The demonstration of the migration of talc from the vagina and posterior uterus to the ovary in the rat[J]. Environmental Research. 1986, 40(2),247-250.

5. Johnson Kurt, E., Popratiloff Anastas, Fan Yuwei, McDonald Sandra, Godleski John, J. Analytic comparison of talc in commercially available body powder and in pelvic tissues resected from ovarian carcinoma patients[J]. Gynecologic Oncology.2020,159(2),527-533

6. Wei Han, Lin Cong, Peng Chen. Preliminary Discussion of the Detection Method for Asbestos[J]. Key Engineering Materials.2017,4348,80-84.

7. Rustin, G. J., Nelstrop, A. E., Bentzen, S. M., Bond, S. J., McClean, P. Selection of active drugs for ovarian cancer based on CA-125 and standard response rates in phase II trials.[J]. Journal of clinical oncology : official journal of the American Society of Clinical Oncology.2000,18(8),1733-1735.

8. Jiwen Wang et al. Diagnostic accuracy of serum HE4, CA125 and ROMA in patients with ovarian cancer: a meta-analysis[J]. Tumor Biology.2014,35(6), 6127-6138.

9. Yuhuan Shen, Li Zhao, Shiming Lu. Diagnostic performance of HE4 and ROMA among Chinese women[J]. Clinica Chimica Acta.2020,500,42-46.

10. Kim Yong Man et al. Evaluation of the accuracy of serum human epididymis protein 4 in combination with CA125 for detecting ovarian cancer: a prospective case-control study in a Korean population.[J]. Clinical Chemistry And Laboratory Medicine.2011,49(3),527-34.

11. ZhiMin Lu, Dai Yin. Characteristics of infant skin structure and design of cosmetics[J]. China Cleaning Industry.2020,6,124-128.

12. Gao Yin, Lu Nan, LeiLei Zhi, LiXiao Jiang, XiaoYan Liu.Research progress of skin structure and physiological characteristics in infants[J]. Chinese Journal Of Aesthetic Medicine.2015,24(03),77-80.

13. Stamatas, G. N. et al.Infant skin microstructure assessed in vivo differsfrom adult skin in organization and at thecellular level [J]. Pediatr Dermatol2010,27(2),125-131.

14. Onder, Yumrutas, Murat, Kara, Remzi, Atilgan, Salih Burcin, Kavak, Ibrahim, Bozgeyik, Ekrem, Sapmaz.Application of talcum powder, trichloroacetic acid and silver nitrate in female rats for nonsurgical sterilization: evaluation of the apoptotic pathway mRNA and miRNA genes.[J].International Journal Of Experimental pathology.2015,96(2),111-5.

15. Hamilton, T. C. et al. Effects of talc on the rat ovary.[J]. British Journal Of Experimental Pathology. 1984, 65(1),101-106.

16. Nicole, M. et al. Molecular Basis Supporting the Association of Talcum Powder Use With Increased Risk of Ovarian Cancer[J]. Reproductive Sciences.2019,26(12),1603-1612. 
17. Wentzensen Nicolas,O'Brien Katie M. Talc, body powder, and ovarian cancer: A summary of the epidemiologic evidence.[J]. Gynecologic Oncology.2021.

18. Berge Wera, Mundt Kenneth, Luu Hung, Boffetta Paolo. Genital use of talc and risk of ovarian cancer: a meta-analysis.[J]. European journal of cancer prevention : the official journal of the European Cancer Prevention Organisation (ECP) .2018,27(3),248-257.

19. Ross Penninkilampi, Guy, D., Eslick. Perineal Talc Use and Ovarian Cancer: A Systematic Review and Meta-Analysis[J]. Epidemiology.2018,29(1),41-49.

\section{Figures}
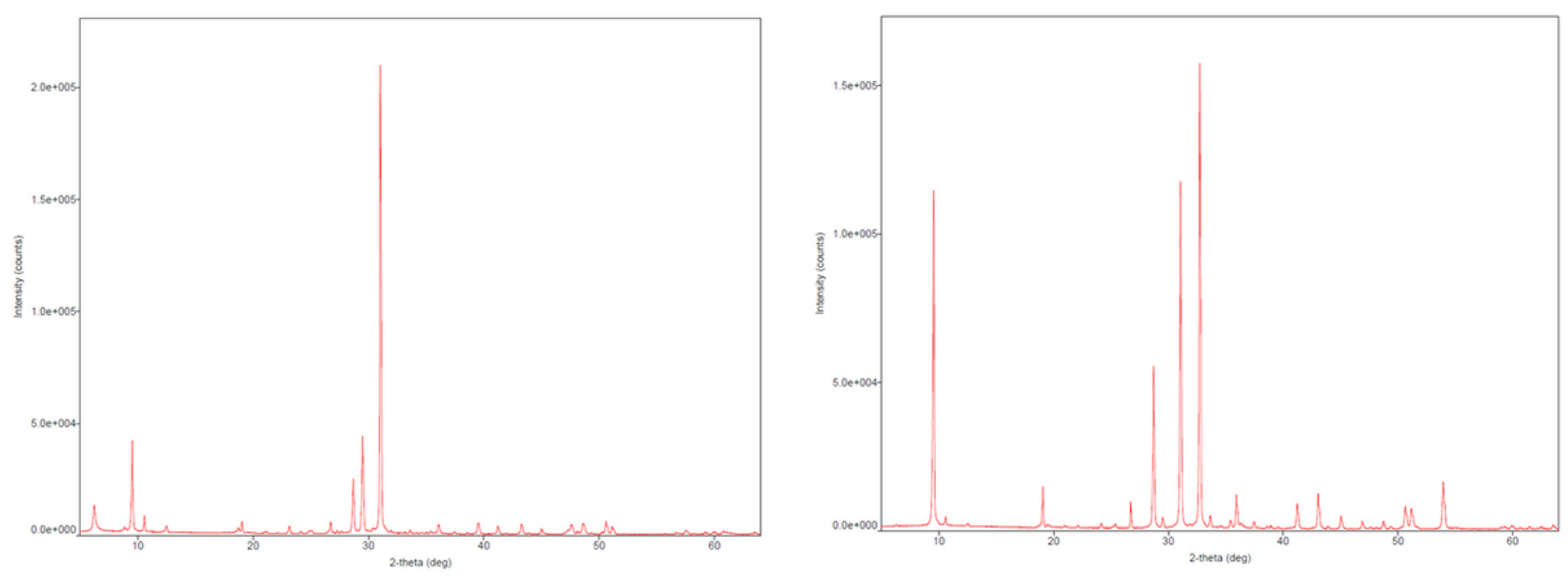

Figure 1

X-ray diffraction patterns of asbestos-containing body powder
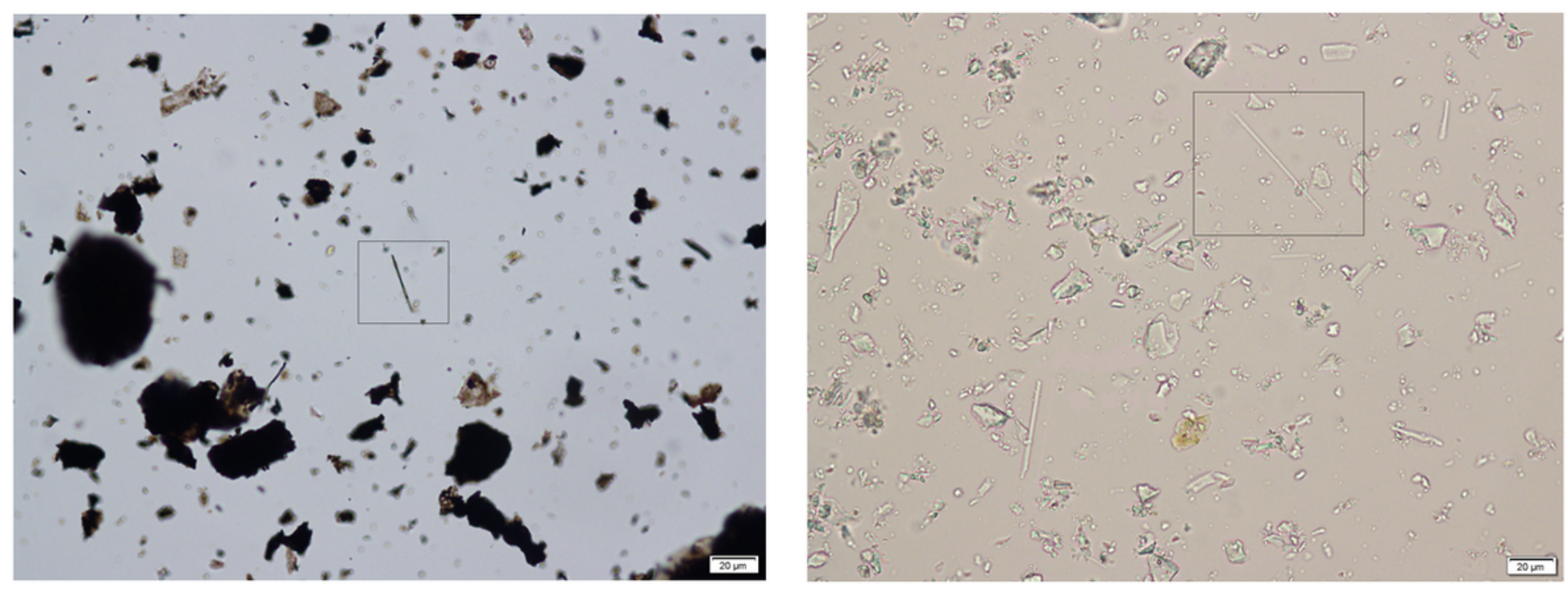

Figure 2 

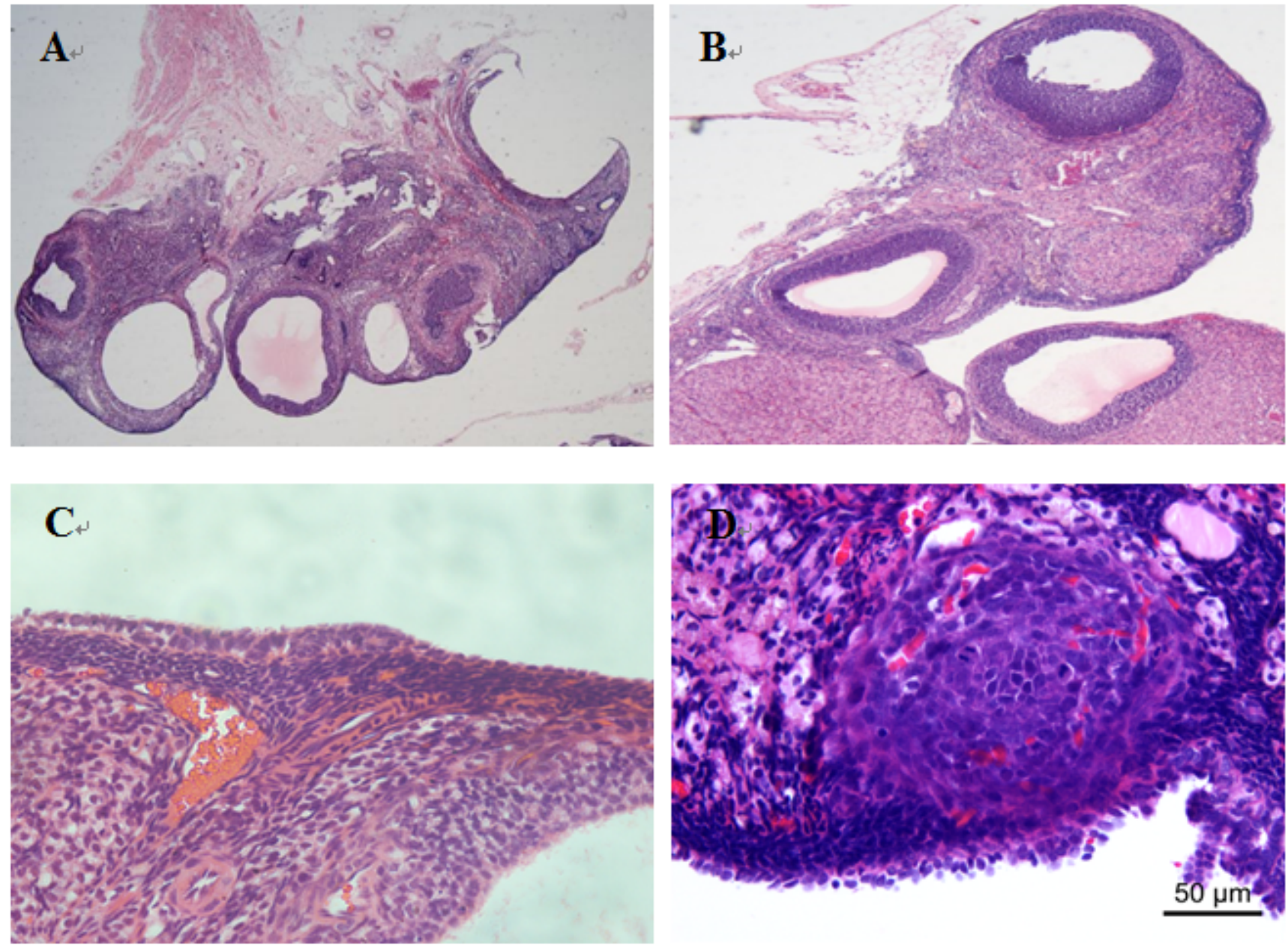

\section{Figure 3}

Morphological changes of ovarian tissue in SD rats. A, control group

(original magnification $\times 40$ ), B, talc group (original magnification $\times 40$ ), C, asbestos group (original magnification $\times 400$ ), D, asbestos+talc group (original magnification $\times 400$ ).

\section{Supplementary Files}

This is a list of supplementary files associated with this preprint. Click to download.

- rawdate.xls 